\title{
Telepathology in Norway: A Review Article
}

\author{
Aleksandar Vodovnik ${ }^{\mathbf{1}}$ and Mohammad Reza F Aghdam ${ }^{2 *}$ \\ ${ }^{1}$ Department of Pathology, Førde Central Hospital, Førde, Norway \\ ${ }^{2}$ Landmo Nursing Home and Rehabilitation Center, Nordre Land Municipality, Norway
}

*Corresponding author: Mohammad Reza F Aghdam, Landmo Nursing Home and Rehabilitation Center, Nordre Land Municipality, Norway

ARTICLE INFO

Received: 幽 April 12, 2021

Published: 幽 April 27, 2021

Citation: Aleksandar Vodovnik and Mohammad Reza F Aghdam. Telepathology in Norway: A Review Article. Biomed J Sci \& Tech Res 35(2)-2021. BJSTR. MS.ID.005687.

Keywords: Telepathology; Digital Pathology; Norway
ABSTRACT

Introduction: We reviewed a development and status of telepathology in Norway.

Methods: The article is based on search in PubMed including "digital pathology" and "Norway", "digital pathology" or "telepathology" and "Norway", and "telepathology" and "Norway" as search terms.

Results: Our review of selected 42 articles, published in peer reviewed journals, showed a steady development of telepathology and digital pathology in Norway contributing to widely accepted general applications and routine diagnostic uses including digital image analysis.

Conclusions: The organization and execution of pathology services in histology, cytology and autopsy including teaching, research, and interdepartmental collaboration have been all improved by implementation of telepathology and digital pathology in Norway.

Abbreviations: NST: Norwegian Centre for Telemedicine; OSODs: Original Second Opinion Diagnoses; DIA: Digital Image Analysis; M-CSF: Macrophage Colony-Stimulating Factor; CSF-1R: Colony-Stimulating Factor-1 Receptor; TGFbeta: Transforming Growth Factor-Beta; COX-2: Cyclooxygenase-2; MAI: Mitotic Activity Index; PC: Perivascular Cells; TAM: Tumor-Associated Macrophages; PR: Progesterone Receptor; miHC: Multiplex Immunohistochemistry; ER: Estrogen

\section{Introduction}

Telepathology, also known today as digital pathology, whole slide imaging and virtual microscopy, stands for diagnostics, education and research using digital images of microscopic slides. Since the pioneering work of Weinstein RS, telepathology has become an integral part of a modern pathology diagnostics. In addition to better ergonomics, instant access to slides and remote diagnostics, considerable technological advances contributed to the growing appeal of telepathology through years. Those evolved from still image diagnostics, through robotic microscopy and hybrid systems to novel scanning techniques [1-3]. Nordrum I and Eide TJ introduced telepathology in Norway in the late 1980 [4-9]. Multiple Norwegian pathology departments have been since implementing telepathology solutions in diverse clinical settings and degrees [10-17]. The Norwegian Centre for Telemedicine (NST) has made a tremendous contribution to the development and implementation of telemedicine and health services in Norway, including telepathology, over the past three decades. It has served as a WHO Collaboration Center for telemedicine since 2002. Since its introduction in 1996, an official telemedicine fee schedule has made telemedical services reimbursable by national health insurers for the first time in the world [18]. In this article, we reviewed development and status of telepathology in Norway, based of works published in peer reviewed journals. The list of reviewed articles in chronological order is shown in (Table 1). 
Table 1: Chronological list of reviewed article on telepathology in Norway.

\begin{tabular}{|c|c|c|c|}
\hline Authors & $\begin{array}{l}\text { Publication } \\
\text { Year }\end{array}$ & $\begin{array}{l}\text { Reference } \\
\text { Number }\end{array}$ & Subject \\
\hline Nordrum I, et al. [4] & 1991 & 4 & Remote frozen section service, telepathology project in northern Norway \\
\hline Eide T], et al. [5] & 1991 & 5 & Use of telecommunications in pathology and anatomy services \\
\hline Eide T], et al. [6] & 1994 & 6 & Current status of telepathology \\
\hline Kayser K [19] & 1995 & 19 & Telepathology in Europe, practical use \\
\hline Nordrum I, et al. [23] & 1995 & 23 & Remote frozen section service in Norway \\
\hline Nordrum I [7] & 1996 & 7 & Telepathology, is there a future \\
\hline Elford DR [10] & 1997 & 10 & Telemedicine in northern Norway \\
\hline Nordrum I, et al. [8] & 1997 & 8 & Breast carcinoma diagnosed by telepathology \\
\hline Nordrum I [9] & 1998 & 9 & Real-time diagnoses in telepathology \\
\hline Haugen OA, et al. [11] & 1999 & 11 & Telepathology in mid-Norway \\
\hline Abeler VM, et al. [12] & 1999 & 12 & Telepathology in Norway \\
\hline Knudsen P, et al. [24] & 2000 & 24 & Telepathology at Norwegian Radium Hospital \\
\hline Nordrum I, et al. [25] & 2000 & 25 & Inaccuracy about telepathology from Radium hospital \\
\hline Bjørnsson BL, et al. [26] & 2000 & 26 & Telepathology in Norwegian Radium hospital \\
\hline Farstad IN, et al. [13] & 2000 & 13 & Pathology, changing discipline \\
\hline Aas IH [20] & 2002 & 20 & Telemedicine and changes in the distribution of tasks between levels of care \\
\hline Reith A [14] & 2002 & 14 & Experience with use of telemedicine in Norway \\
\hline Obstfelder A [21] & 2003 & 21 & Social dilemmas in the implementation of the Norwegian Pathology Network \\
\hline Majak BM [15] & 2004 & 15 & Experience from use of telepathology in Telemark \\
\hline Nordrum I, et al. [27] & 2004 & 27 & Diagnostic accuracy of second-opinion diagnoses based on still images \\
\hline Hartvigsen G, et al. [18] & 2007 & 18 & Challenges in telemedicine and eHealth \\
\hline Norum J, et al. [22] & 2007 & 22 & Prioritisation of telemedicine services for large scale implementation in Norway \\
\hline Skaland I, et al. [31] & 2008 & 31 & Subjective and digital image analysis of HER2/neu expression scores in breast cancer \\
\hline Richardsen E, et al. [32] & 2008 & 32 & Prognostic impact of M-CSF, CSF-1 receptor, CD68 and CD3 in prostatic carcinoma \\
\hline Skaland I, et al. [33] & 2009 & 33 & Validating prognostic value of proliferation measured by РРНЗ \\
\hline Richardsen E, et al. [34] & 2010 & 34 & COX-2 is overexpressed in primary prostate cancer with metastatic potential \\
\hline Gudlaugsson E, et al. [35] & 2012 & 35 & Effects of different techniques for measurement of Ki67 proliferation in breast cancer \\
\hline Nielsen B, et al. [36] & 2012 & 36 & Automatic segmentation of cell nuclei in Feulgen-stained sections of prostate cancer \\
\hline Gudlaugsson E, et al. [37] & 2013 & 37 & Comparison of prognostic factors in breast cancer \\
\hline Vodovnik A [28] & 2015 & 28 & Distance reporting in digital pathology \\
\hline Richardsen E, et al. [38] & 2015 & 38 & M-CSF1 predicts breast cancer progression and mortality \\
\hline Vodovnik A [29] & 2016 & 29 & Diagnostic time in digital pathology \\
\hline Mezheyeuski A, et al. [39] & 2016 & 39 & $\begin{array}{c}\text { Survival-associated heterogeneity of marker-defined perivascular cells in colorectal } \\
\text { cancer }\end{array}$ \\
\hline Richardsen E, et al. [40] & 2017 & 40 & Evaluation of proliferation marker Ki-67 in large prostatectomy cohort \\
\hline Hagland HR, et al. [41] & 2017 & 41 & Blood T-cells and Intratumoural Density and Location of T-Cells in Colorectal Cancer \\
\hline Nastic D, et al. [42] & 2017 & 42 & $\begin{array}{c}\text { Selective marker panel increases reproducibility and accuracy in endometrial } \\
\text { diagnosis }\end{array}$ \\
\hline Danielsen HE, et al. [43] & 2017 & 43 & Prognostic markers for colorectal cancer - estimating ploidy and stroma \\
\hline Bengtsson E, et al. [16] & 2017 & 16 & Computer-aided diagnostics in digital pathology. \\
\hline Vodovnik A, et al. [17] & 2018 & 17 & Complete routine remote digital pathology services \\
\hline Vodovnik A, et al. [30] & 2018 & 30 & Remote autopsy services \\
\hline Lopes N, et al. [44] & 2020 & 44 & Digital image analysis of multiplex fluorescence IHC in colorectal cancer \\
\hline
\end{tabular}




\section{Methods}

We based our review on the search conducted in PubMed. Search terms included "digital pathology" and "Norway", "digital pathology" or "telepathology" and "Norway", and "telepathology" and "Norway". We have used a combination of "MeSH" and "All field" options to conduct the search. Inclusion criteria were all papers published to the present day. We have found altogether 211 publications in all three searches, cross-matched them, and ended up with 41 publications fulfilling our subject criteria.

\section{Discussion}

\section{General Considerations}

The term "telepathology" has been used in 1986 for the first time. Initially, dynamic (real time) and static image (store-and-forward) telepathology were the first two competing modalities. The hybrid imaging system, which combined these two primary pathology imaging modalities into a single multi-modality pathology imaging system was established by Norwegian laboratory in 1989. Similar hybrid systems followed in other countries as well. It is important to emphasize that hybrid telepathology systems were the starting point for the first genuinely sustainable telepathology services. Since then, astounding technical progress resulted in development "virtual microscopy" telepathology (also called "whole slide image" telepathology or "WSI" telepathology of digital pathology). WSI eventually became the preferred digital telepathology digital imaging choice. Recurrent interest in dynamic-robotic telepathology was partly related to concerns over the lack of Z-axis focusing capabilities of the early WSI processors. Two original U.S. patents for robotic telepathology (issued in 1993 and 1994) expired in 2010, which has stimulated many digital pathology equipment companies to incorporate dynamic-robotic telepathology advances into new WSI products. The dynamic-robotic telepathology module offered a solution to the up-and-down focusing issue. WSI and dynamic robotic telepathology are now being used in the new class of imaging system called the "WSI-enhanced dynamic robotic telepathology system".

Major manufacturers of WSI processor equipment companies have adopted this approach and developed WSI-enhanced dynamicrobotic digital telepathology systems, marketed under different labels. Successful marketing of these systems may help overcome the resistance of some pathologists to immerse digital pathology into their routine pathology laboratories. These advanced telepathology systems could play a crucial role in provisions of complete pathology services to the majority of world's underserved population for the foreseeable future. At the same time, this approach could finally enable the delivery of renowned patientcentered healthcare in the future [1,3]. Advancement of pathology relates to implementation of novel biological techniques and influence of electronic equipment devices, which influence transfer of information and images. The rise in complexity in disease classification influences specific needs for data exchange and expert consultation. Routine applications of telecommunications in pathology have been influenced nearly exclusively through the use of public systems, including intraoperative evaluation of histological images through frozen section service, second opinion consultations, panel discussions, stereological techniques, and access to the storage of digital images. The remote control of microscopes diagnostic purpose has worked successfully from Norway and Switzerland. Diagnostic information and microscopic images were transferred using satellite communication in Norway. In Switzerland, the ISDN technology was, however, in use.

Those experiences showed that remotely controlled microscopes are practical and reliable for routine diagnostic services in pathology. They have also established that the image quality was adequate for complex diagnoses. For qualified consultation, landline phones were used in lung pathology, where their impact on the diagnostic turnaround could not be underestimated. Diagnostic accuracy and consistency, state of the art staining and tissue handling, shown improvements besides the positive legal aspects [19]. The University Hospital of Troms $\varnothing$ was a hub for diverse telemedicine activities since the late 1980 s. Those included teleradiology, telepathology, tele dermatology, remote endoscopy, remote gastroscopy, tele echocardiography, transmission of electrocardiograms, telepsychiatry, electronic delivery of laboratory results, and distance learning for health professionals. As a result, the Department of Telemedicine in Troms $\varnothing$ has been designated the national centre of competence in telemedicine since 1993. Due to growing knowledge in the field, increasing number of health-care professionals exposed to and using telemedicine in the routine fashion, continued sustenance of the Norwegian health authorities, a national center guiding telemedicine research, no licensing barriers within the country, nationwide ISDN and reimbursement for telemedicine services, the future of telemedicine in Norway looked promising at the time [10].

The investigation by Aas IH, which was part of a larger study, investigated possible changes in distribution of tasks between general practitioners and specialists and between general and university hospitals. They have carried out qualitative interviews with 30 persons involved in tele dermatology, tele-otolaryngology, telepsychiatry, and a telepathology frozen-section service in Norway. The results indicated that telemedicine does not produce large changes in the distribution of tasks. The reported effects were largest and most complex for telepsychiatry, followed by teledermatology. Local variations in how telemedicine is practiced may explain the variation in the findings between telemedicine applications [20]. Another study explored the implementation of the Norwegian Pathology Network (PatNet). The study included fourteen departments of pathology from the university and local hospitals that subscribed to PatNet. Techniques used in sociological 
research were applied in an attempt to identify potential obstacles to implementation of telemedicine regarding coordination and cooperation. The implementation of PatNet has not accomplished this to a sufficient degree. The pioneers of PatNet expected that easy access to the network combined with comprehensive information and discussion within the network would attract users. Further and detailed information and discussions needed to be produced by users in order to attract further users, in expectation that the network was to be run by itself.

As this did not work, the study suggested that an open discussion among pathologists would be useful to explore and highlight emerging dilemmas [21]. The Northern Norway Regional Health Authority requested the evaluation of telemedicine services in the region in 2005, in order to define their suitability for a largescale implementation. Of 282 reports included in the study, apart from projects not focusing on secondary health care, 46 studies representing 21 topics entered the final analysis. The analysis involved a self-developed scoring tool. Eleven topics were chosen for large scale implementation and grouped according to priority. The first priority topics were teleradiology, digitalization of patient records and education. The second priority topics were teledialysis, pre-hospital thrombolysis, telepsychiatry and teledermatology. The third priority topics were pediatrics, district medical centers, tele-ophthalmology, and tele-otorhinolaryngology. No priority was designed for the projects in cardiology, endocrinology, geriatrics, gynecology/obstetrics, pathology, and nursing/care. Support services, training, research, financial incentives, and interaction between clinicians and information and communication personnel were put forward as important in motivating health-care professional to use telemedicine [22].

\section{Diagnostic Considerations (Frozen Section, Routine Histology, Cytology, And Autopsy, Second Opinion)}

Telepathology comprises the transmission of microscopic images via the telecommunications network. These include teleconferences discussing histologic slides from the university hospital with clinicians at local hospitals. A frozen section service to Kirkenes Hospital, based on remote control of a robotic microscope and transmission of high-quality video images of the slide, has also been successfully established [5]. Remote frozen section services were offered using a remote control of a motorized video-microscope at the local hospital, more than $400 \mathrm{~km}$ from the workstation at the university hospital. The frozen section images were transmitted via a two-way telephone and video network (2 Mbit/s capacity). The pathologists diagnosed the images on computer screens. The study included 17 patients. The remote diagnoses and final diagnoses on formalin-fixed and paraffin-embedded material conformed in all cases. The average examination time for frozen section was 15 minutes (range, 5 to 30 minutes). The interpretation of the slides was deemed difficult due to deficient quality of the video images in none of the cases. For hospitals without local pathology services or with shortages in subspecialist, telepathology solutions were deemed to be a worthwhile substitute [4]. Two local hospitals in northern Norway were equipped with a motorized video microscope, remotely controlled from the workstation at the university hospital. The video images were transmitted through two-way audio and video network.

This system utilized compressed images with $384 \mathrm{Kbit} / \mathrm{s}$ transfer rates. The video images of surgical specimens and frozen sections were displayed on monitors at both sites. In nearly five years, tissue from 100 patients have been examined. Breasts and thyroid tissues accounted for 74 of cases. The digital and final diagnoses conformed in 89 cases. There were one false positive, three false negative and seven deferred diagnosis regarding malignancy. The false positive diagnosis had no clinical implications. Two of the deferred diagnosis turned out to be malignant. The average examination time of frozen sections was 12 minutes (range, 3 to $45 \mathrm{~min}$ ). This concept of remote frozen section services was at the time recommended for hospitals without a local service in diagnostic pathology [23]. Pathologists at the university hospital used a dynamic-robotic system (Telemed A200, AM Elektronikk A/S), for three years in 1995 for 116 diagnostic sessions towards two rural Norwegian hospitals. In $90 \%$ of the cases a diagnosis was provided. No false positive diagnosis was made with only $3 \%$ false negative cases, with no clinical implications. Cases were deferred cases $(10 \%)$ mainly due to poor quality of the frozen sections and pathologist's conservative attitudes. The diagnostic accuracy was good, but for safety reasons regular telepathology should be offered, to keep up the skills of technicians and pathologists involved in the service. In Mid-Norway, five more hospitals were afterwards included in the network comprising of eight hospitals in the region [11].

Pathologists in Telemark have used the telepathology for teaching cytopathology to cytoscreeners and pathology assistants. One was local and the other remote, in the refional hospital from their experience at the time, it was more convenient and cheaper to use internet-based than Path Sight telepathology [15]. Knudsen, et al. [24] reviewed the telepathology activity at the Norwegian Radium Hospital between 1994 and 2000, including aspects of their functionality. Frozen section diagnosis was provided on 74 slides using two different telepathology systems. One system was used to measure its functionality for second opinion services. A new Internet-based system was developed to provide additional functionalities. A telepathology system with a digital camera outperformed the one with an analog camera regarding a diagnostic accuracy. Authors found out that the precision of telepathology service was determined by image quality. Telepathology was subsequently deemed feasible for second opinion pathology services [24-26]. Second opinion pathology services are one 
of the important routines. At the time, second opinion services outside the hospital were contracted through sending still images via an electronic network. This study aimed to examine levels of diagnostic accuracy for second opinion diagnostics based on still images. These were selected from 90 archived cases originally referred for second opinion.

Two pathologists made diagnosis first on the still images (phase 1) followed by glass slides (phase 2). Both diagnoses were compared with the original second opinion diagnoses (OSODs). The same diagnostic was made on both still images and glass slides. There were 29 cases with the diagnosis discordant with the OSOD. Incorrect benign diagnoses were recorded in three cases. Eight cases showed incorrect malignant diagnoses. Eight diagnoses were false-negative and six false-positive regarding malignancy. Eleven diagnoses had potential clinical implications. Authors acknowledged the importance of observer variability in diagnostic histopathology in general. The results supported the routine use of still images for second opinion diagnostic services [27]. Department of Pathology in Førde has conducted a digital pathology project in 2011. They addressed all aspects of routine pathologist workload, resulting in ten studies within the project. Vodovnik A has shown that much of the digitalization could be achieved using existing IT solutions at institutions, supporting efficient and reliable remote services for the core pathology services in histology and cytology. The most important contributors to the success of remote diagnostics services were stable network speeds, fully integrated laboratory information management system, technical reliability, working flexibility, larger displays, and shorter turnaround.

A further expansion of our remote pathology services, diagnostic and educational, rely on gaining experience in digital reporting and marginal investment in information technology. The conclusion was that the full benefit of successful establishment of digital pathology services could be achieved through the diverse adaptations to structuring of pathology services [28]. Further studies showed that shorter diagnostic turnaround could be achieved using digital pathology. Stable network speeds, fully integrated LIMS, and double displays contributed the most, but better ergonomics, larger viewing field, and absence of physical slide handling were also deemed important factors in achieving shorter diagnostic and non-diagnostic time. Study design, image size, number of cases, specimen type, network speed, and participant's level of confidence and experience in digital reporting were important differences against other studies. Technical and ergonomic improvements in working stations and increased experience in digital reporting have a potential to further improve diagnostic time and broaden routine requisition of digital pathology [29]. Vodovnik, et al. [30] also showed the feasibility of autopsy services when involving experienced staff and existing IT solutions. This had a positive effect on flexibility and interest of autopsy technicians in autopsy services [30].
Department of Pathology in Førde have achieved complete routine remote digital pathology services in 2015. The implementation of digital pathology has advanced allocations and management of facilities in histology, cytology, and autopsy including both local and regional education and cooperation [17].

\section{Digital Image Analysis}

Skaland, et al. [31] correlated modified FISH scores with HER2 $3+$ score, agreed between pathologists, and digital image analysis (DIA) scores. Modified FISH were shown better than conventional FISH scores. DIA scoring of HER2 was deemed as a superior to the manual scoring [31]. Macrophage colony-stimulating factor (M-CSF) connects to colony-stimulating factor-1 receptor (CSF1R) stimulating proliferation and differentiation of monocytes, macrophages, and their bone marrow progenitors. Studies shown the increase of M-CSF, CSF-1R, CD68, and CD3 in variety of human malignancies. Their prognostic value in primary prostatic cancer has been studied but not defined with certainty. Authors aimed to compare M-CSF, CSF-1R, CD68 and CD3 expressions in both metastatic and non-metastatic prostatic cancer. DIA analysis of malignant tumor and its stroma was conducted on 59 cancer specimens. 32 of those were patients with metastases and 27 patients without metastatic disease. The expression of abovementioned marker played a remarkable role in prognostication of metastatic disease in prostatic carcinomas [32]. Skaland, et al. [31] conducted a study on prognostic value of the proliferation marker phosphohistone H3 (PPH3). The study included 241 breast cancer patients under 71 years of age, who had a long-term follow-up and received no adjuvant treatment. DIA was used to measure PPH3 expression. Of thirty-seven patients with metastatic disease 29 died.

The prognostic threshold of PHH3 was the strongest prognostic factor. It has been shown as a robust, highly reproducible, prognostic over the thresholds around 13, and is the overall strongest prognostic factor amongst studied for patients with invasive, lymph node negative, and under 71 years old [33]. Immune modulating molecules cyclooxygenase-2 (COX-2), transforming growth factor-beta (TGF-beta) and interleukin-10 (IL-10) regulate advancement of cancer cells, with conflicting data on their exact role in prostate cancer. Authors compared their expression in patients with a prostate cancer, irrespective of metastatic disease status. They have also included Ki67 to measure tumours proliferation fraction. DIA from stromal and epithelial parts of the tumor tissue from 59 patients. COX-2, TGF-beta and Ki67 showed stronger tumor expression in metastatic than cancers without metastatic disease. Higher COX-2 tumor reactivity was associated with death from prostate cancer when univariate analysis was used. In other markers no association with death was found [34]. Ki67 proliferation fraction was found to have a prognostic value in breast cancer. However, techniques to measure its expression in cancer differed between laboratories. Authors conducted a study 
on reproducibility and prognostic value of different methods to sample and measurement of Ki67. 237 breast cancer patients, without adjuvant therapy, were standardized by section thickness, automated antigen retrieval and immunohistochemistry.

Ki67 value was assessed using rapid estimation, square guided count, computerized point-grid-sampling interactive morphometry (CIM), and automated DIA. Ki67 DIA evaluation was the best prognostic factor, even if its threshold varied (4-15\%). The best area to prognosticate Ki67 was tumor's periphery. This study cast doubts on treatment guidelines when subjective counting methods are used to evaluate Ki67 expression [35].

Nuclear DIA has been used to gather a quantitative information for the cancer diagnosis and prognostication, but dependable automated nuclear count solutions limited its high-volume analysis. Authors have developed a methodology for nuclear segmentation sections from prostatic cancer stained by Feulgen stain. They combined a local adaptive threshold identification and object perimeter gradient verification to detect the nuclei followed by with active contour model, featuring optimized initialization. Manual and automatic markings from 30 images and altogether 924 nuclei were compared. The success of automatic markings was not significantly different from the manual but paved the way for large-scale studies in this regard [36]. The mitotic activity index (MAI), PPH3 and Ki67 play a role in prognostication of early breast cancer. However, the effect of their independency from each other as well as other variables have not been studied.

Authors conducted a study on breast cancer patients without previous adjuvant therapy measuring MAI, Ki67, PPH3, estrogen (ER) and progesterone receptor (PR), HER2, cytokeratin 5/6, and cytokeratin 14 expressions on DIA in peripheral areas of tumor samples. The comparison showed addition values of Ki67 to PPH3 regarding prognosis, which was not the case for other variables regarding a combination of Ki67 and PHH3. Interestingly, performing PPH3 instead of MAI did not change the value of prognosis. Furthermore, in patients with an early breast cancer, Ki67 evaluated on DIA at 6.5\% value, was a strong prognostic factor. PPH3/Ki67 or MAI/Ki67 were shown as superior prognostic marker [37]. Richardsen, et al. [34] showed that a progression of breast cancers was related to higher reactivity of CSF1, CSF1R, tumorassociated macrophages (TAM), and CD3. CSF1 expression in tumor epithelium was a predictor of mortality in breast cancer [38]. A role of perivascular cells (PC) in a regulation of metastatic potential and cellular immunity has been assumed. A new methodology for DIA analysis of vessels and PC in colonic cancer with metastases was presented in this study. At first, a subset of PC was identified using PDGFR- $\beta$ and/or $\alpha$-SMA immunohistochemistry and showed no relation to vessel's size or density. Additional analyses suggested certain mutations in tumor cells as surrogates of PC status. DIA discovered a significance of associations between lower reactivity of perivascular PDGFR- $\alpha$ and $-\beta$ and shortened survival.
Those two markers were still found unrestrained variables for survival in metastatic colon cancers. This study detected vasculature and oncogene status as decisive of perivascular features in tumors [39]. High reactivity of Ki-67 in prostatic cancer was identified as autonomous negative variable for biochemical failure. Findings from this study were based on visual and DIA scoring systems and promoted Ki-67 as an important, poor prognostic marker in patients with a prostate cancer [40]. Hagland, et al. [41] have pointed out an interaction between CD3 and CD4 positive cells in blood and T-cells from the edge and center of tumor in samples from patients with a colorectal cancer, through DIA [41]. In the study by Nastic, et al. [42] endometrial carcinoma samples were additionally investigated using a panel of ER, PR, P53, and DNA ploidy. Twelve gynecologic pathologists from four Nordic countries diagnosed case without and with the marker panel. Authors found an increase in interobserver agreement from $75.8 \%$ to $84 \%$ with the panel of markers. This was further improved on hysterectomy samples, from $83.6 \%$ to $88.7 \%$ [42]. Danielsen, et al. [43] measured a value of ploidy and DIA morphometry, as a prognostic variable, on samples from 2624 colorectal cancer with early disease. They identified that a combination of ploidy and stroma to tumor ratio, detects colorectal cancer patients with low, intermediate, or high risk of the disease specific death. This could also effectively stratify a subpopulation of patients with a characteristic risk of recurrence and assist the choice of adjuvant therapy for these patients [43].

In the study by Lopes, et al. [44] flourescence-based multiplex immunohistochemistry (mIHC) was used to measure CDX2, SOX2, SOX9, E-cadherin, and $\beta$-catenin in patients with a colorectal cancer. The results were collated with a standard IHC performed on the same tissue. The methods were comparable. Although DIA refined levels of differentiation in cases with high and low reactivity, there were challenges in DIA interpretation of membrane reactions, more consistently evaluated on the microscope. Combined use of DIA and mIHC assured quantification rates of marker's expression. The result suggested a strong negative correlation between CDX2 and SOX2. Prognostication levels of CDX2 were confirmed, however, visualization and detection of associations between pathology and biology of tumors. This method showed as a potential novel way to regiment CDX2 detection [44].

\section{Conclusion}

Since the pioneering work of Nordrum and Eide, telepathology in Norway has come a long way in the last 30 years, improving routine diagnostics, education, and consultations. There is ongoing national digital pathology project, which has been driven by continuous increase in workload and shortages of pathologists. Main objectives with the project are to improve patient care through shorter turnaround time, faster and more precise diagnostics, increase patient safety, increase utilization of the existing overall expertise and capacity of the pathology departments, strengthen education, and maintain recruitment to the pathology profession. 
Its concept was introduced in 2016 and business plan approved in 2017. The project has entered its implementation phase in 2018. The investment is expected to be repaid by the end of 2022 . All four regional health authorities are expected to become fully digitalized by 2025 (https://ehelse.no/prosjekt/digital-patologi).

\section{Acknowledgement}

We are grateful to our institutions for their support. The authors declare no conflicts of interest.

\section{References}

1. Weinstein RS, Graham AR, Lian F, Braunhut BL, Barker GR, et al. (2012) Reconciliation of diverse telepathology system designs. Historic issues and implications for emerging markets and new applications. APMIS 120: 256-275.

2. Farahani N, Riben M, Evans AJ, Pantanowitz L (2016) International Telepathology: Promises and Pitfalls. Pathobiology 83(2-3): 121-126.

3. Weinstein RS, Holcomb MJ, Krupinski EA (2019) Invention and Early History of Telepathology (1985-2000). J Pathol Inform 10: 1.

4. Nordrum I, Engum B, Rinde E, Finseth A, Ericsson H, et al. (1991) Remote frozen section service: A telepathology project in northern Norway. Hum Pathol 22: 514-518.

5. Eide T], Nordrum I, Engum B, Rinde E (1991) [Use of telecommunications in pathology and anatomy services]. Tidsskr Nor Laegeforen 111: 17-19.

6. Eide TJ, Nordrum I (1994) Current status of telepathology. APMIS 102: 881-890.

7. Nordrum I (1996) Telepathology. Is there a future? Telemed Today 4: 24-26.

8. Nordrum I, Isaksen V, Arvola L (1997) Breast carcinoma diagnosed by telepathology. J Telemed Telecare 3: 172-173.

9. Nordrum I (1998) Real-time diagnoses in telepathology. Adv Clin Path 2: $127-131$

10. Elford DR (1997) Telemedicine in northern Norway. J Telemed Telecare $3: 1-22$.

11. Haugen OA, Halvorsen TB, Aarset H, Ovrehus TA, Torp SH, et al. (1999) Telepathology in Mid-Norway. Tidsskr Nor Laegeforen 119: 355-358.

12. Abeler VM, Danielsen HE (1999) Telepathology in Norway. Tidsskr Nor Laegeforen 119: 1335.

13. Farstad IN, Bofin A, Goplen AK, Kjellevold KH, Nordrum I, et al. (2000) [Pathology--a changing discipline]. Tidsskr Nor Laegeforen 120: 17961801

14. Reith A (2002) Experience with the use of telemedicine in Norway. Int Comput Dent 5: 115-117.

15. Majak BM (2004) Experience from the use of telepathology in Telemark. Histopathology 44: 82.

16. Bengtsson E, Danielsen H, Treanor D, Gurcan MN, MacAulay C, et al. (2017) Computer-aided diagnostics in digital pathology. Cytometry A 91: 551-554.

17. Vodovnik A, Aghdam MRF (2018) Complete Routine Remote Digital Pathology Services. J Pathol Inform 9: 36.

18. Hartvigsen G, Johansen MA, Hasvold P, Bellika JG, Arsand E, et al. (2007) Challenges in telemedicine and eHealth: Lessons learned from 20 years with telemedicine in Tromsø. Stud Health Technol Inform 129: 82-86.

19. Kayser K (1995) Telepathology in Europe. Its practical use. Arch Anat Cytol Pathol 43(3): 196-199.
20. Aas IH (2002) Telemedicine and changes in the distribution of tasks between levels of care. J Telemed Telecare 8(2): 1-2.

21. Obstfelder A (2003) Social dilemmas in a telemedicine network: Experience with the implementation of the Norwegian Pathology Network. J Telemed Telecare 9(1): S29-30.

22. Norum J, Pedersen S, Størmer J, Rumpsfeld M, Stormo A, et al. (2007) Prioritisation of telemedicine services for large scale implementation in Norway. J Telemed Telecare 13: 185-192.

23. Nordrum I, Eide TJ (1995) Remote frozen section service in Norway. Arch Anat Cytol Pathol 43: 253-256.

24. Knudsen P, Ryther AJ, Nesheim JA, Abeler VM, Nesland JM, et al. (2000) [Telepathology at the Norwegian Radium Hospital]. Tidsskr Nor Laegeforen 120: 2170-2173.

25. Nordrum I, Isaksen V, Johansen M, Strand V (2000) [Inaccuracy about telepathology from the Radiumhospital]. Tidsskr Nor Laegeforen 120: 2939.

26. Bjørnsson BL (2000) Telepathology in the Norwegian Radiumhospital. Tidsskr Nor Laegeforen 120: 2579.

27. Nordrum I, Johansen M, Amin A, Isaksen V, Ludvigsen JA (2004) Diagnostic accuracy of second-opinion diagnoses based on still images. Hum Pathol 35: 129-135.

28. Vodovnik A (2015) Distance reporting in digital pathology: A study on 950 cases. J Pathol Inform 6: 18.

29. Vodovnik A (2016) Diagnostic time in digital pathology: A comparative study on 400 cases. J Pathol Inform 7: 4.

30. Vodovnik A, Aghdam MRF, Espedal DG (2018) Remote autopsy services: A feasibility study on nine cases. J Telemed Telecare 24: 460-464.

31. Skaland I, Øvestad I, Janssen EA, Klos J, Kjellevold KH, et al. (2008) Comparing subjective and digital image analysis HER2/neu expression scores with conventional and modified FISH scores in breast cancer. J Clin Pathol 61: 68-71.

32. Richardsen E, Uglehus RD, Due J, Busch C, Busund LT (2008) The prognostic impact of M-CSF, CSF-1 receptor, CD68 and CD3 in prostatic carcinoma. Histopathology 53: 30-38.

33. Skaland I, Janssen EA, Gudlaugsson E, Klos J, Kjellevold KH, et al (2009) Validating the prognostic value of proliferation measured by Phosphohistone $\mathrm{H} 3$ (PPH3) in invasive lymph node-negative breast cancer patients less than 71 years of age. Breast Cancer Res Treat 114: $39-45$.

34. Richardsen E, Uglehus RD, Due J, Busch C, Busund LT (2010) COX-2 is overexpressed in primary prostate cancer with metastatic potential and may predict survival. A comparison study between COX-2, TGF-beta, IL10 and Ki67. Cancer Epidemiol 34: 316-322.

35. Gudlaugsson E, Skaland I, Janssen EA, Smaaland R, Shao Z, et al. (2012) Comparison of the effect of different techniques for measurement of Ki67 proliferation on reproducibility and prognosis prediction accuracy in breast cancer. Histopathology 61(6): 1134-1344.

36. Nielsen B, Albregtsen F, Danielsen HE (2012) Automatic segmentation of cell nuclei in Feulgen-stained histological sections of prostate cancer and quantitative evaluation of segmentation results. Cytometry A 81: 588-601.

37. Gudlaugsson E, Klos J, Skaland I, Janssen EA, Smaaland R, et al. (2013) Prognostic comparison of the proliferation markers (mitotic activity index, phosphohistone H3, Ki67), steroid receptors, HER2, high molecular weight cytokeratins and classical prognostic factors in $\mathrm{T}_{1-2} \mathrm{~N}_{0} \mathrm{M}_{0}$ breast cancer. Pol J Pathol 64: 1-8.

38. Richardsen E, Uglehus RD, Johnsen SH, Busund LT (2015) Macrophagecolony stimulating factor (CSF1) predicts breast cancer progression and mortality. Anticancer Res 35: 865-874. 
39. Mezheyeuski A, Bradic Lindh M, Guren TK, Dragomir A, Pfeiffer P, et al. (2016) Survival-associated heterogeneity of marker-defined perivascular cells in colorectal cancer. Oncotarget 7(29): 41948-41958.

40. Richardsen E, Andersen S, Al Saad S, Rakaee M, Nordby Y, et al. (2017) Evaluation of the proliferation marker Ki-67 in a large prostatectomy cohort. PLoS One 12(11): e0186852.

41. Hagland HR, Lea D, Watson MM, Søreide K (2017) Correlation of Blood T-Cells to Intratumoural Density and Location of CD3(+) and CD8(+) T-Cells in Colorectal Cancer. Anticancer Res 37: 675-683.

42. Nastic D, Shanwell E, Wallin KL, Valla M, Måsbäck A, et al. (2017) A Selective Biomarker Panel Increases the Reproducibility and the Accuracy in Endometrial Biopsy Diagnosis. Int J Gynecol Pathol 36: 339347.

ISSN: 2574-1241

DOI: 10.26717/BJSTR.2021.35.005687

Mohammad Reza FA. Biomed J Sci \& Tech Res

(C) (P) This work is licensed under Creative

Submission Link: https://biomedres.us/submit-manuscript.php
43. Danielsen HE, Hveem TS, Domingo E, Pradhan M, Kleppe A, et al. (2018) Prognostic markers for colorectal cancer: Estimating ploidy and stroma. Ann Oncol 29: 616-623.

44. Lopes N, Bergsland CH, Bjørnslett M, Pellinen T, Svindland A, et al. (2020) Digital image analysis of multiplex fluorescence IHC in colorectal cancer recognizes the prognostic value of CDX2 and its negative correlation with SOX2. Lab Invest 100: 120-134.

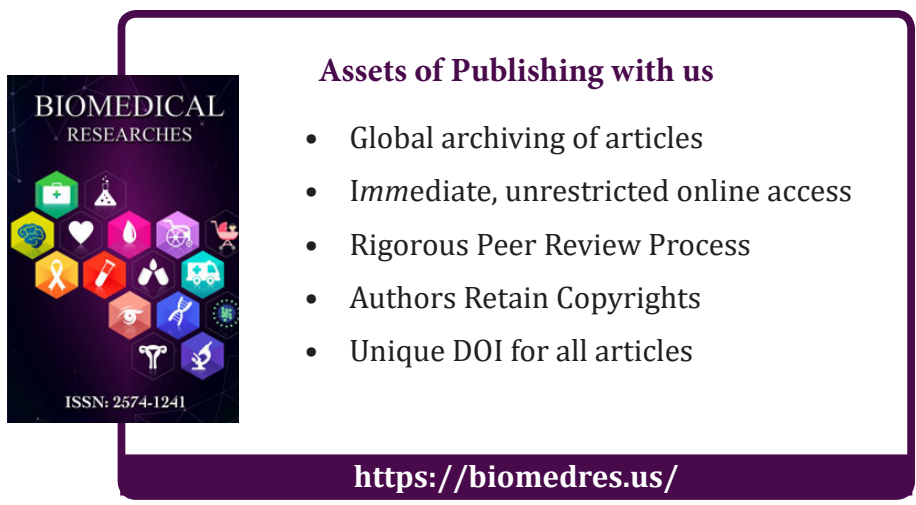

